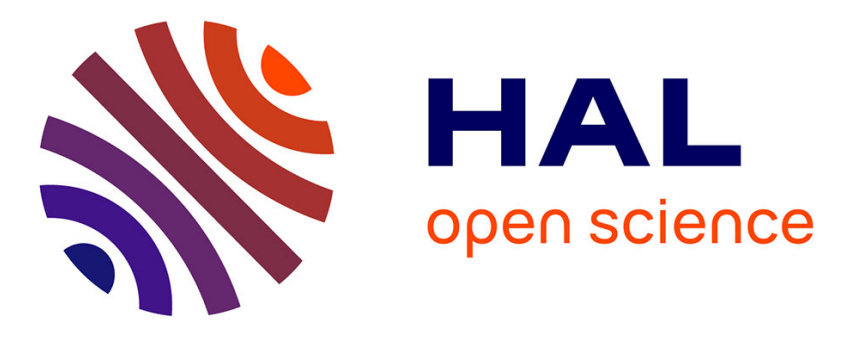

\title{
Janus hybrid silica/polymer nanoparticles as effective compatibilizing agents for polystyrene/polyamide-6 melted blends
}

T. Parpaite, B. Otazaghine, A. S. Caro-Bretelle, A. Taguet, R. Sonnier, J. M. Lopez-Cuesta

\section{To cite this version:}

T. Parpaite, B. Otazaghine, A. S. Caro-Bretelle, A. Taguet, R. Sonnier, et al.. Janus hybrid silica/polymer nanoparticles as effective compatibilizing agents for polystyrene/polyamide- 6 melted blends. Polymer, 2016, 90, pp.34-44. 10.1016/j.polymer.2016.02.044 . hal-02906427

\section{HAL Id: hal-02906427 \\ https://hal.science/hal-02906427}

Submitted on 7 Jan 2021

HAL is a multi-disciplinary open access archive for the deposit and dissemination of scientific research documents, whether they are published or not. The documents may come from teaching and research institutions in France or abroad, or from public or private research centers.
L'archive ouverte pluridisciplinaire HAL, est destinée au dépôt et à la diffusion de documents scientifiques de niveau recherche, publiés ou non, émanant des établissements d'enseignement et de recherche français ou étrangers, des laboratoires publics ou privés. 


\title{
Janus hybrid silica/polymer nanoparticles as effective compatibilizing agents for polystyrene/polyamide- 6 melted blends
}

\author{
T. Parpaite, B. Otazaghine*, A.S. Caro, A. Taguet, R. Sonnier, J.M. Lopez-Cuesta \\ Centre des Matériaux des mines d'Alès, 6, avenue de Clavières, 30319 Alès, France
}

\begin{abstract}
A B S T R A C T
Silica/polystyrene Janus hybrid nanoparticles (JHNPs) were synthesized at the gram scale via mini-emulsion polymerization technique and then incorporated into polystyrene/polyamide- 6 melted blends. Due to their intrinsic amphiphilic properties, the JHNPs migrate to the interface of the PS/PA6 blend during the mixing process and play the role of a compatibilizing agent. First, a significant decrease of the size of the dispersed nodules was observed even at low content of JHNPs, whereas an optimum content of JHNPs corresponding to the saturation of the polystyrene/polyamide-6 (PS/PA6) interface was found in the 3-5 phr range. Secondly, the rheological properties of the blends were also significantly modified. The complex viscosity increased as a function of JHNPs content over the entire frequency range studied. Up to 5 phr of JHNPs, a high increase of the viscosity at low frequencies was observed. This is attributed to the formation of a solid network through the matrix/nodules interface of the nanocomposite. Finally, the Palierne model extended to the field of immiscible polymer blends was used; an important decrease of the effective interfacial tension of the system was observed when JHNPs were added. The asymmetry of these nanoparticles allows the combination of a "Pickering effect" of a solid particle with a "copolymer effect" of an amphiphilic structure. The synthesis procedure of JHNPs developed in this work renders these silica/ polymer JHNPs interesting as a route for the compatibilization of polymer blends.
\end{abstract}

Keywords:

Janus nanoparticles

Compatibilization

Polymer blends

Interfacial tension

Linear viscoelasticity

Palierne model

\section{Introduction}

Blending polymers is an efficient and versatile way to cumulate properties of materials and has proven to be industrially relevant. However, due to immiscibility between most polymers, it is necessary to improve interfacial adhesion between the polymeric components by compatibilization. Compatibilization solutions usually range from reactive polymers, organic copolymers (block, random or grafted) [1], $\gamma$-irradiation or electron beam [2], cosolvent or ionic interaction, and nanoparticles [3-6]. It is now well-established that nanoparticles with different aspect ratio and chemical nature, that could reduce the interfacial tension between the two separated polymers can compatibilize polymer blends [4]. Nanoparticles can migrate at the interface of a polymer blend for thermodynamic reasons and that can be predicted by calculating the wetting parameter [6,7]. Recently, a new class of nanoparticles with an amphiphilic anisotropic structure comprising two distinct hemispheres has emerged: the Janus nanoparticles (JNPs). Organic

\footnotetext{
* Corresponding author.

E-mail address: belkacem.otazaghine@mines-ales.fr (B. Otazaghine).
}

$[3,8,9]$ or hybrid $[10,11]$ JNPs can be tailored to achieve the compatibility of two polymer phases. Walther et al. [3] were the first to evaluate the ability of JPNs to compatibilize polymer blends. They chose organic JNPs based on polystyrene-block-polybutadiene-block-poly(methyl methacrylate) triblock terpolymer (SBM) for the compatibilization of polystyrene/poly(methyl methacrylate) (PS/PMMA) blends. Those SBM JNPs were obtained by a multiple-step process consisting of the synthesis of the triblock polymer via anionic polymerization followed by the crosslinking of the polybutadiene core of SBM lamella. They clearly showed that the Janus SBM segregate at the interface of a PS/PMMA blend and exhibit superior compatibilization abilities compared to conventional linear SBM copolymer. Weiss et al. [10] performed anisotropic modifications of kaolinite platelets to obtain Janus kaolinite particles. They showed also for these Janus particles a localization at the interface of PS/PMMA blends obtained by a solvent casting procedure. More recently, Bahrami et al. [8] investigated the compatibilizing effect of SBM JNPs in poly(2,6-dimethyl-1,4-phenylene ether)/poly(styrene-co-acrylonitrile) (PPE/SAN) blends using both lab-scale and industrial scale melt processing. The authors showed similar results i.e. selective adsorption of JNPs at the interface of 
polymer blends and superior interfacial activities in terms of droplets size reduction compared to conventional compatibilizers.

The use of compatibilizers in blends facilitates the droplets breakup and allows the coalescence inhibition of the dispersed phase and the reduction of the interfacial tension. One of the most efficient way to prove the compatibilization of a polymer blend is the measurement of an interfacial tension decrease [12]. Various techniques have been used to measure the interfacial tension between two polymers. Most methods are based on drop profiles such as the pendant drop, the breaking thread, the imbedded fiber retraction and the retraction of deformed drop [12,13]. These methods require long experimental times, involve complicated experimental procedures and cannot be used for all polymers (the three latter methods are possible only for transparent polymers in molten state). Moreover these tests consider only one drop, thread or fiber. Several experiments have to be repeated to obtain an accurate value of the interfacial tension. A few methods are based on rheological measurements [13]. These methods afford the advantage of testing the total interface in the blend and giving a more representative value. It is known that relaxation times of drops in immiscible molten polymers are increased compared to pure components. Rheological measurements allow the evaluation of these relaxation times. Especially, models were developed to deduce the interfacial tension from rheological measurements [14-16]. In the 1990s Palierne [17] proposed a model of blend linear viscoelasticity based on two hypothesis: both polymers are Newtonian in their molten state and dispersed phase nodules are and remain spherical during the process. The Palierne's model describes the frequency $\left(w=\left(w_{i}\right)_{\mathrm{i}=1 . . \mathrm{n}}\right)$ dependence of complex modulus $\left(\mathrm{G}^{*}\right)$ of viscoelastic emulsions under small amplitude oscillatory flow as a function of the complex moduli of both phases $\left(G_{m}^{*}\right.$ for the matrix and $\mathrm{G}_{\mathrm{d}}^{*}$ for the droplets phases), the ratio of the interfacial tension (denoted $\alpha$ ) and the droplet size when the polydispersity of nodules distribution is low (i.e. the ratio between the average and the median diameters is lower than 2). Many authors proposed to qualify successfully the miscibility degree of various polymers via this model (PS/PMMA [18-20], PS/polyethylene (PE) [21], polyethylene terephthalate glycol/polyester-ethylene vinylacetate (PETG/EVA) [22]). When nodules are not monodisperse, Palierne's model could take into account the polydispersity of nodule sizes introducing the volume fraction $\phi_{\mathrm{i}}$ associated to each class of nodule diameter $D_{i}$; the equations are modified as follows:

$G^{*}(w)=G^{\prime}(w)+i G^{\prime \prime}(w)=G_{m}^{*}(w)\left[\frac{1+3 \sum_{i} \Phi_{i} H_{m}^{*}(w)}{1-2 \sum_{i} \Phi_{i} H_{m}^{*}(w)}\right]$

where polymer blends by this way considering a polydisperse distribution of nodules sizes [23-26]. In the case of polymer phases exhibiting a high viscosity ratio (for example highly viscous disperse phase), modeling difficulties could arise from low moduli measurements obtained at low frequencies [23]. In the case of filled polymer blends the Palierne's model needs to be modified. Indeed, Elias et al. [27] calculated an apparent interfacial tension considering the partition of silica between dispersed and matrix phases, using TEM and SEM observations. They further [15] used Kerner model [28] and Taylor equation $[29,30]$ to confirm the dispersed phase size reduction and to prove the decrease of the apparent interfacial tension when 3 wt\% of hydrophilic and hydrophobic silica NPs are added into PP/EVA blends.

In the first part of this study JHNPs were prepared by a waterbased miniemulsion polymerization process. Then, after their characterization, these anisotropic nanoparticles were incorporated by melt blending into a PS/PA6 80/20 blend. The emulsification role of these JHNPs was investigated and the compatibilizing effect was evaluated by the measurement of the interfacial tension using rheological analysis.

\section{Materials and methods}

\subsection{Materials}

CRISTAL 1340 polystyrene (PS) was purchased from Total Petrochemicals (Feluy, Belgium). It is a crystal PS with a high molecular weight, a melt flow index (MFI) of $4 \mathrm{~g} .10 \mathrm{~min}^{-1}$ (with $5 \mathrm{~kg}$ at $200{ }^{\circ} \mathrm{C}$ ) and a density of $1.05 \mathrm{~g} / \mathrm{cm}^{3}$. Aquamid AQL40 polyamide-6 (PA6) was supplied by Aquafil Technopolymers (Arco, Italy). Its melting point temperature is $220{ }^{\circ} \mathrm{C}$ (ISO Test Method 3146), its MFI is $160 \mathrm{~g} .10 \mathrm{~min}^{-1}$ (with $5 \mathrm{~kg}$ at $275^{\circ} \mathrm{C}$ ) and its density is $1.14 \mathrm{~g} / \mathrm{cm}^{3}$. Polymer pellets were dried under vacuum at $80{ }^{\circ} \mathrm{C}$ for $12 \mathrm{~h}$ before processing.

Tetraethoxysilane (TEOS, 99.9\%) and sodium dodecyl sulfate (SDS, 99,9\%) were purchased from Rectapur. Ethanol (EtOH, 96\%), ammonia $\left(\mathrm{NH}_{3}, 28 \%\right)$ hexadecane (HD), 2,2azobis(isobutyronitrile) (AIBN) and 3methacryloxypropyltrimethoxysilane (MPS, 99.9\%) were purchased from Sigma-Aldrich, Merck, Prolabo, Fluka and, Wackers Silicon, respectively. All reactants were used as received.

\subsection{Synthesis of Janus hybrid silica/PS nanoparticles}

In a one-necked round-bottom flask $(250 \mathrm{~mL}) \quad 2 \mathrm{~g}$ $\left(9.6 \times 10^{-3} \mathrm{~mol}\right)$ of TEOS, $0.5 \mathrm{~g}\left(2.0 \times 10^{-2} \mathrm{~mol}\right)$ of $3-$ methacryloxypropyltrimethoxysilane (MPS) and $0.4 \mathrm{~g}$ $\left(1.8 \times 10^{-2} \mathrm{~mol}\right)$ of hexadecane (HD) were introduced. This mixture was considered as the organic phase. Then $40 \mathrm{~mL}$ of a sodium

$H_{i}^{*}(w)=\frac{\frac{4 \sigma}{D_{i}}\left(2 G_{m}^{*}(w)+5 G_{d}^{*}(w)\right)+\left(G_{d}^{*}(w)-G_{m}^{*}(w)\right)\left(16 G_{m}^{*}(w)+19 G_{d}^{*}(w)\right)}{\frac{40 \sigma}{D_{i}}\left(G_{m}^{*}(w)+G_{d}^{*}(w)\right)+\left(2 G_{d}^{*}(w)+3 G_{m}^{*}(w)\right)\left(16 G_{m}^{*}(w)+19 G_{d}^{*}(w)\right)}$

For a narrow nodule dispersion, Eqs. (1) and (2) can be simplified into: $\forall i, D_{i}=D_{v}, \Phi_{i}=\Phi$ with $D_{v}$ the volume average diameter and $\Phi$ the dispersed volume fraction rate.

Many authors successfully evaluated the interfacial tension in dodecyl sulfate (SDS) solution ( $1 \mathrm{mM}$ in water) considered as the water phase was added. The organic and water phases were first pre-emulsified by mechanical stirring for 15 min and then treated with ultrasonication for 5 min ( 10 cycles of 30 s of ultrasonication and 30 s of rest) at $120 \mathrm{~W}$ to generate a stable miniemulsion. The $\mathrm{pH}$ of the obtained miniemulsion was adjusted to 9.0 using a $\mathrm{NaOH}$ 
solution $(0.01 \mathrm{M})$. The flask was then equipped with a reflux condenser and the solution heated under stirring for $2 \mathrm{~h}$ at $60{ }^{\circ} \mathrm{C}$. After reaction, the solution was cooled at room temperature and a mixture of $4 \mathrm{~g}\left(3.8 \times 10^{-2} \mathrm{~mol}\right)$ of styrene and $0.08 \mathrm{~g}$ $\left(4.9 \times 10^{-4} \mathrm{~mol}\right)$ of 2,2-azobis(isobutyronitrile) (AIBN) was added. The solution was stirred for $2 \mathrm{~h}$ at room temperature and then heated at $80^{\circ} \mathrm{C}$ for $4 \mathrm{~h}$ to undergo the styrene polymerization. After polymerization, the solution was dried using a freeze drying apparatus. The obtained white powder was washed three times with methanol to remove SDS and three times with cyclohexane to remove HD. Centrifugation was used to remove the solvent between each washing. The obtained product was finally dried under vacuum.

\subsection{Solvent casting method and compounding procedure}

Nanocomposites PS/PA6/JHNPs were prepared by adding 1.5, 3 , 5 and 10 per hundred rate (phr) of Janus hybrid nanoparticles. To avoid handling of dry nanoparticles powders, we used in a first step a solvent casting method to embed Janus nanoparticles into PS film. $1 \mathrm{~g}$ of PS pellets was dissolved in $10 \mathrm{~mL}$ of THF and mixed with desired quantities of JHNPs at room temperature during $12 \mathrm{~h}$ under magnetic stirring. The solvent was then evaporated at room temperature and the obtained film was dried under vacuum at $80^{\circ} \mathrm{C}$ for $3 \mathrm{~h}$. Concerning the compounding procedure, the PS/PA6 weight ratio was fixed at 80/20. During the first minute of mixing, PS pellets are first introduced followed by the PS film containing JHNPs, and finally PA6 pellets. Then, the mixing of the ternary blends is carried out during $4 \mathrm{~min}$ at $250{ }^{\circ} \mathrm{C}$ under nitrogen flow to avoid thermal degradation with a constant screw speed of $120 \mathrm{rpm}$.

\subsection{Samples injection procedure}

At the end of the compounding procedure, nanocomposites are cut in pellets and dry under vacuum at $80{ }^{\circ} \mathrm{C}$ for $3 \mathrm{~h}$ before injection. Then, pellets are directly introduced into the barrel of the mini-injection machine. Barrel and mold temperatures were $240{ }^{\circ} \mathrm{C}$ and $80^{\circ} \mathrm{C}$, respectively. Both injection and holding pressures were set to 3.5 bars. After $10 \mathrm{~s}$ of holding time, the sample was ejected manually by opening the laboratory mold.

\subsection{Rheological investigations}

The rheological properties of the blends were evaluated by oscillatory characterization. All experiments were carried out at $230{ }^{\circ} \mathrm{C}$ under nitrogen flow to avoid thermal degradation. The storage $G^{\prime}$, the loss $G^{\prime \prime}$ moduli and the complex viscosity $\eta^{*}$ of the samples were measured with constant strain oscillatory deformation of $3 \%$ within the linearity zone for all blends. A frequency scan from $w=\left(w_{i}\right)_{\mathrm{i}=1.29}=100-0.01 \mathrm{rad} / \mathrm{s}$ was applied. A strain sweep was applied to fix the strain limit for the linear viscoelastic response.

\subsection{Optimization method}

Let $G_{i \exp }^{\prime}$ and $G_{i \exp }^{\prime \prime}$ the vector values of the storage and loss moduli at each frequency. The corresponding numerical values, $G^{\prime}\left(\alpha, G_{m}^{*}, G_{d}^{*}\right)$ and $G^{\prime \prime}\left(\alpha, G_{m}^{*}, G_{d}^{*}\right)$ which depend on the interfacial tension and the complex shear moduli of the matrix and the dispersed phase, are computed from eqs. (1) and (2). The gap between experimental and numerical values is computed via a cost function (denoted $\mathrm{J}$ ) written as the squared 2-norm:
$J(\alpha)=\sum_{i=1}^{n} \frac{\left(G_{i}^{\prime}(\alpha)-G_{i \exp }^{\prime}\right)^{2}}{\left(G_{i \exp }^{\prime}\right)^{2}}+\frac{\left(G_{i}^{\prime \prime}(\alpha)-G_{i \exp }^{\prime \prime}\right)^{2}}{\left(G_{i \exp }^{\prime \prime}\right)^{2}}$

Hence, the interfacial tension $\bar{\alpha}$ is the solution of the minimization problem given by

$\min J(\alpha)$

$\alpha \geq 0$

Equation (4) has been solved using Matlab ${ }^{\circledR}$ software with a constrained Levenberg-Marquardt algorithm which is rather classical in the literature for solving identification problem.

\subsection{Instrumentation}

\subsubsection{Dynamic light scattering (DLS)}

A Malvern Nano ZS DLS apparatus was used to determine hydrodynamic diameter (Z-average) and polydispersity index (PDI) of JHNPs. Water was used as dispersant, temperature of measurement was $25^{\circ} \mathrm{C}$, and equilibration time was $120 \mathrm{~s}$. Common values of refractive and absorption indices for silica (1.45 and 0.01, respectively) were used. At least, three measurements were performed for each sample to ensure repeatability.

\subsubsection{Scanning electron microscopy (SEM)}

Blends or extracted nodules were observed with a Scanning Electron Microscope (FEI Quanta 200 ESEM) under high vacuum. Blends were fractured in liquid nitrogen and the fractured surface was observed whereas suspensions of extracted nodules were evaporated on copper grids. Thin layer of carbon was deposited on all samples before observation to enhance conductivity of the sample.

\subsubsection{Chemical analyses via high resolution transmission electron microscopy}

Chemical composition of JHNPs was investigated by an EDX microanalyzer (PGT IMIX PC) mounted on a HRTEM JEOL JEM 2011 equipped with $\mathrm{LaB}_{6}$ filament. Images of samples were collected using a $4008 \times 2672$ pixel CDD camera (Gatan Orius SC1000). One drop of diluted solution (around 100 times) of JHNPs was deposited on carbon film copper grid and then evaporated at room temperature (at least $12 \mathrm{~h}$ ) before observations.

\subsubsection{Twin-screw mini-mixer}

Melt compounding was carried out using a DSM twin-screw mini-extruder. The mixing of the blends is carried out during $4 \mathrm{~min}$ at $250{ }^{\circ} \mathrm{C}$ with a constant screw speed of $120 \mathrm{rpm}$.

\subsubsection{Mini-injection machine}

A mini-injection machine (IM15 from Zamak Mercator) was used to inject disk samples with a thickness of $1.5 \mathrm{~mm}$ and a diameter of $25 \mathrm{~mm}$.

\subsubsection{Laser diffraction particle size analyzer}

A Coulter LS 13230 (Coulter Beckmann Co.) laser diffraction particle size analyzer instrument was used to determine the size distribution of extracted PA6 nodules. The PA6 nodules were collected after dissolving the PS matrix using THF solvent. Size measurements were performed using the micro liquid module (MLM, $15 \mathrm{~mL}$ ) in THF, obscuration was $10 \pm 2 \%$. At least, three measurements were performed for each sample. This protocol has been fully detailed by the team in a previous paper [31]. 


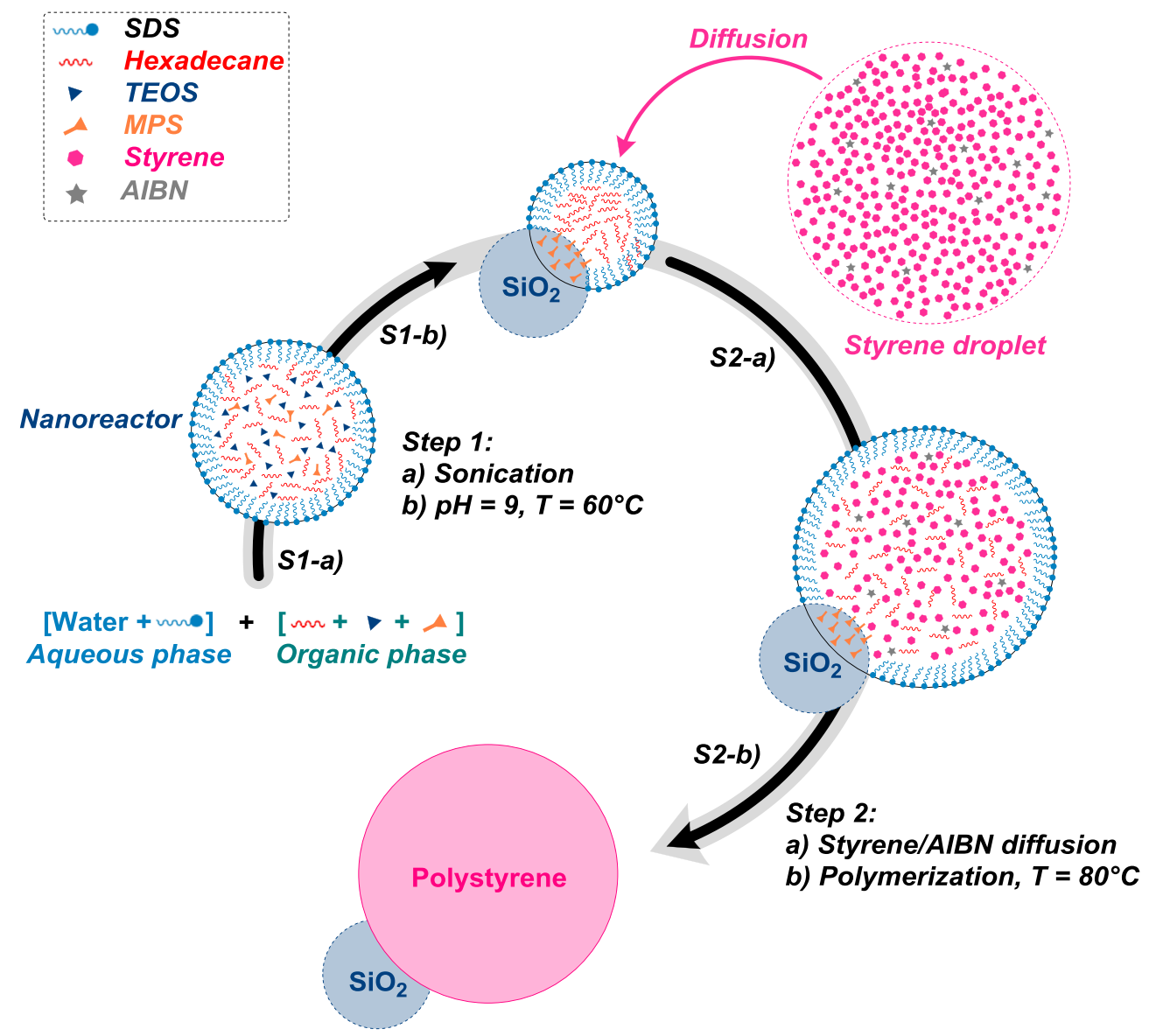

Janus Hybrid Silica/PS Nanoparticle

Fig. 1. Schematic illustration of the "two-step" method used to produce silica/PS JHNPs.

\subsubsection{Rheometer}

A Rheometric Scientific ARES rheometer (TA instrument) equipped with parallel plate geometry (plate diameter $25 \mathrm{~mm}$, gap $1 \mathrm{~mm}$ ) was used to investigated rheological properties of blends.

\section{Results and discussion}

\subsection{Synthesis of Janus hybrid nanoparticles (JHNPs)}

The synthesis of silica/PS Janus hybrid nanoparticles used in this work is based on the work of Lu et al. [32] published in 2008. The authors used a one-step method based on a miniemulsion process to produce asymmetric inorganic-organic dimers composed of one silica $\left(\mathrm{SiO}_{2}\right)$ part and one polystyrene (PS) part. Firstly, silica and polystyrene precursors (tetraethoxysilane and styrene, respectively) compose the initial homogeneous organic phase. Then a phenomenon of internal phase separation which occurs in each droplet of the mixture allows obtaining Janus $\mathrm{PS} / \mathrm{SiO}_{2}$ nanoparticles with snowman-like morphology. The little part of these nanoparticles is silica and the big one is PS. Moreover both moieties were covalently bonded to each other thanks to the use of a coupling agent: Y-methacryloxypropyltrimethoxysilane. The silane part of this agent reacts during the silica formation and the methacrylate function reacts during the styrene polymerization. Using this protocol, the authors announced a yield of synthesis estimated via TEM analysis at about $40 \%$ by volume. To optimize this yield of synthesis, the protocol of Lu et al. [32] was modified to become a two-step process. In our new protocol, styrene is not introduced in the initial reactive solution and the first step concerns only the formation of Janus silica nanoparticles. We assume that even in the absence of styrene, the presence of hexadecane in the initial mixture allows the internal phase separation process described by Lu et al. [32]. For each nanoreactor of the miniemulsion, the first

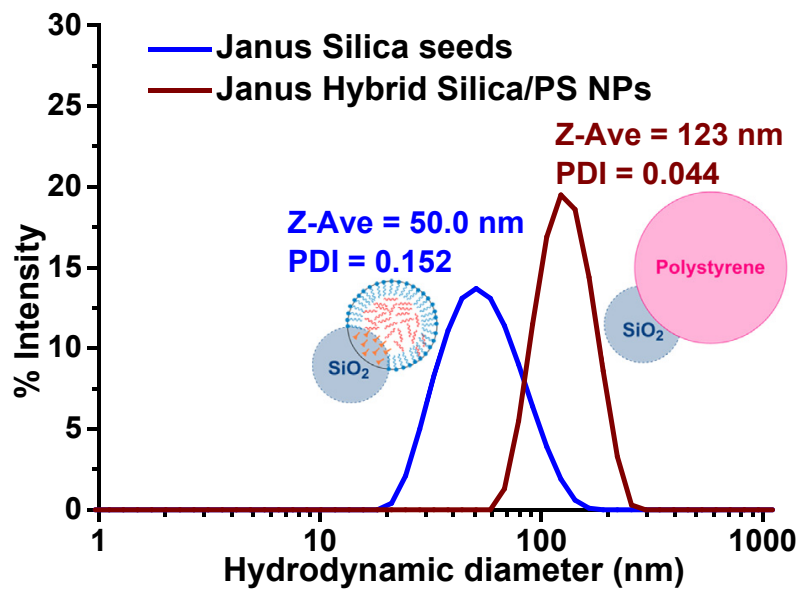

Fig. 2. Size distribution of Janus silica seeds and JHNPs measured by DLS 


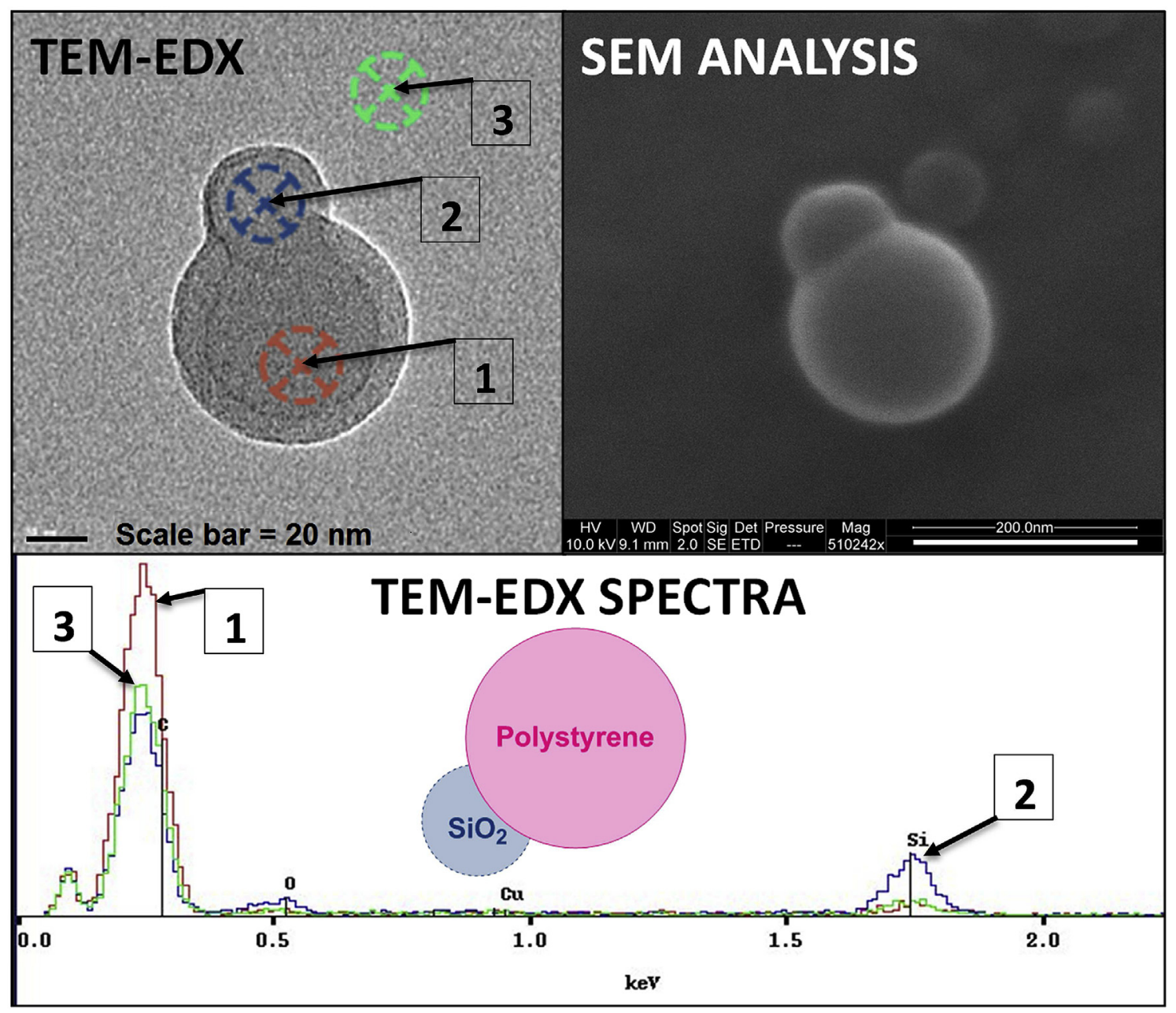

Fig. 3. Evidence of the asymmetric composition of JHNPs via TEM-EDX analysis.

step allows the formation of anisotropic silica nanoparticles with a hemisphere functionalized with methacrylate groups covered with an hexadecane droplet (see step S1 in the Fig. 1). For the second step, styrene is added and the PS moiety is obtained by the swelling of the organic hemisphere by monomers units followed by their polymerization (see step S2 in the Fig. 1). In our system, silica precursor (TEOS), coupling agent (MPS) and co-stabilizer (HD) form the homogeneous organic phase. After sonication, stable oil in water $(\mathrm{O} / \mathrm{W})$ miniemulsion is obtained. Both concentration of surfactant and ultrasonic protocol are adjusted to obtain nanoreactors with mean diameter of around $50 \mathrm{~nm}$. Then, the $\mathrm{pH}$ and the temperature of the media are adjusted to 9 and $60{ }^{\circ} \mathrm{C}$, respectively in order to initiate the internal phase separation process. At $\mathrm{pH} 9$, hydrolysis and polycondensation of TEOS molecules form polar silica network $\left(\mathrm{SiO}_{2}\right)$. In this stage, the initial homogeneity of the nanodroplets is modified: polar silica in one side and apolar hexadecane and organic part of the co-stabilizer in the other side. Due to its amphiphilic structure, the MPS coupling agent self-assembles at the interface between silica and hexadecane (HD) to decrease interfacial tension inside the nanoreactors. At the end of the first step (Fig. 1 S1-b), Janus silica seeds are obtained with a mean hydrodynamic diameter measured by Dynamic Light Scattering (DLS) of around $50 \mathrm{~nm}$ (Fig. 2). The second step is the polymerization of styrene (St). When introduced into the oil in water miniemulsion, the monomer and initiator units diffuse into the organic part of the nanodroplets containing HD and methacrylate groups. The solution is then heated at $80{ }^{\circ} \mathrm{C}$ to initiate the copolymerization between styrene and methacrylate units. At the end of the synthesis, JHNPs silica/PS with snowman-like morphology [33-37] are produced

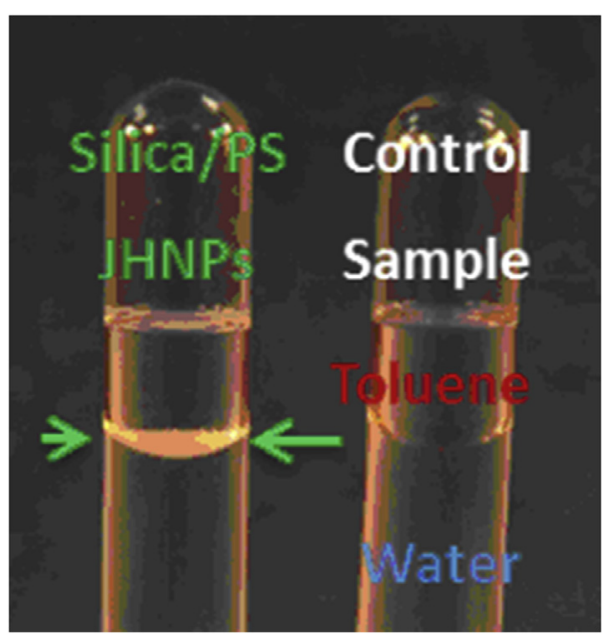

Fig. 4. Photograph of water/toluene biphasic systems (left) with or (right) without JHNPs silica/PS.

Arrows indicate the presence of JHNPs at the solvents interface. 

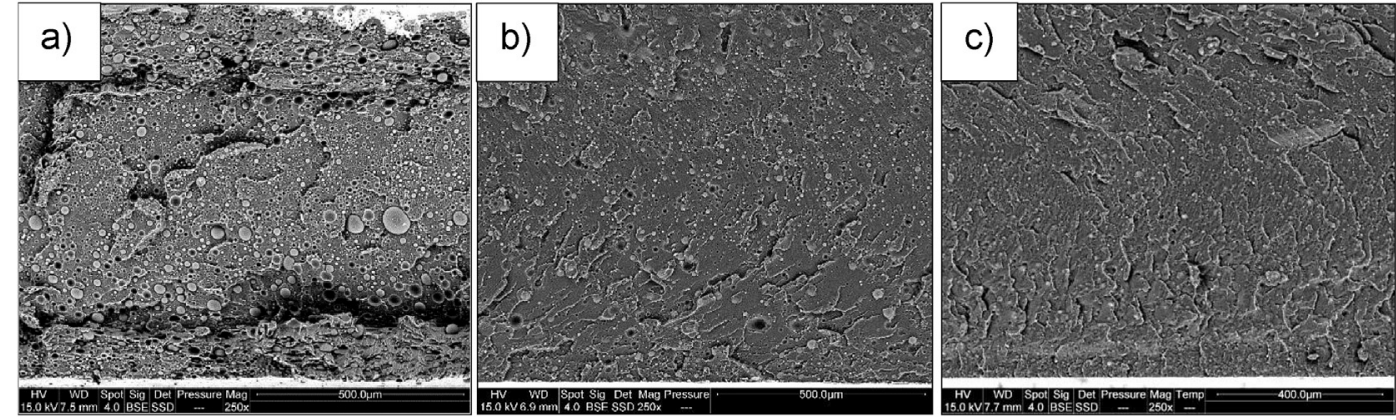

Fig. 5. SEM images of cryo-fractured PS/PA6 80/20 nanocomposites with (a) 0, (b) 1.5 and (c) 3 phr of JHNPs at the end of the rheological test.

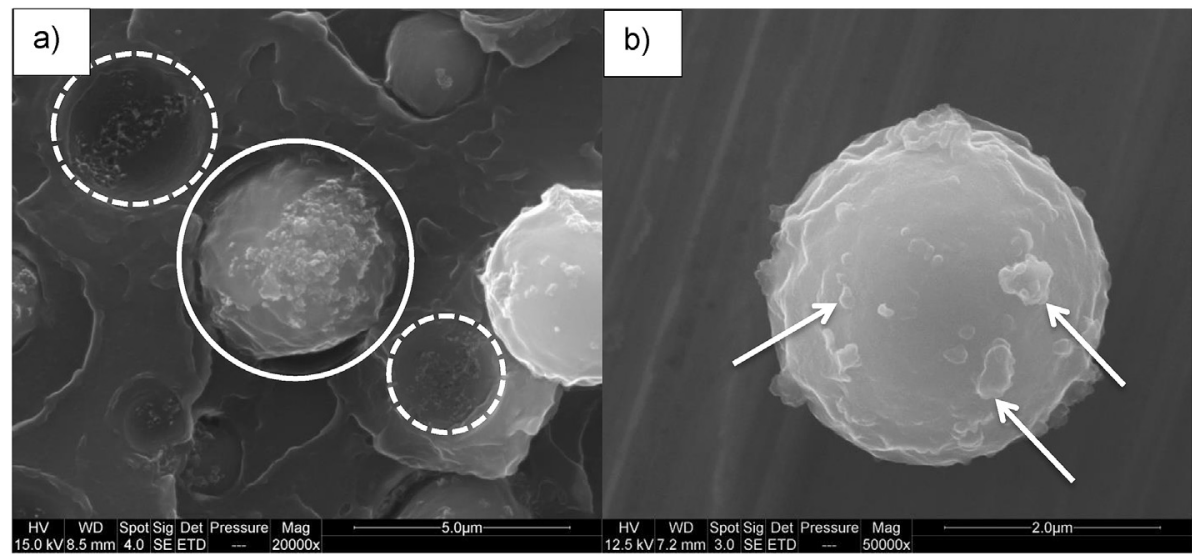

Fig. 6. SEM images of PA6 nodule from PS/PA6/JHNPs 80/20/1.5 system (a) before and (b) after dissolution of PS matrix.

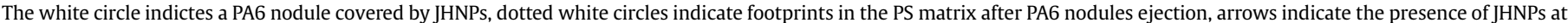
the surface of a PA6 nodule.

with mean diameter of $123 \mathrm{~nm}$ and low polydispersity index (Fig. 2). The internal chemical structure of the resulting JHNPs silica/PS is displayed in Fig. S1. It is important to highlight the key role of the MPS coupling agent that acts as a covalent bridge between the inorganic silica part and the organic PS one. Fig. S2 clearly shows that without MPS (Fig. S2a) no JHNPs are observed by TEM, small silica nanoparticles (blue circles) and PS latex particles (red circles) are isolated. Similar results were obtained by Lu et al. [32] for the one-step method. With small amount of MPS (MPS/TEOS weight ratio equal to 5\%), a few JHNPs appears (see green circles Fig. S2b). Interestingly, some notches (red arrows Fig. S2b) are observed on PS latex particles. These notches indicate the poor adhesion between PS and silica parts due to low concentration in MPS coupling agent [37]. Finally, with optimal concentration of MPS (MPS/TEOS weight ratio equal to 25\%) numerous JHNPs are obtained (green circles Fig. S2c).

\subsection{Evidences of the anisotropic chemical composition of the JHNPS}

To prove the anisotropic chemical character of our JHNPs, TEMEDX analysis was employed. For each JHNP analyzed, three scans were performed: the first one (green spot in Fig. 3) for the background of the observation grid (composed of copper and carbon film), the second one (represented by a blue spot in the Fig. 3) was realized on the smaller part corresponding to the "snowman head" whereas the last one (red spot in Fig. 3) analyzed the bigger part of the nanoparticle corresponding to the "snowman body". For each colored spot the corresponding colored spectrum are also displayed in Fig. 3. The results are similar to those presented by Lu et al. [32] and clearly confirm that the small part is composed essentially of silica whereas the big one is PS. Another method to evaluate the amphiphilic character of such entities is to observe their behavior in polar/apolar immiscible liquids such as water/toluene [38] mixture. When introduced in water/toluene biphasic systems the JHNPs segregate at the interface of both solvents in order to decrease interfacial tensions (Fig. 4). This result confirms also the amphiphilic nature of the synthetized JHNPs. This observation method is purely qualitative, but we assume that it indicates a high yield of functionalization of silica nanoparticles with PS nodules. Indeed, even small quantities of free silica would be enough to cloud the water phase.

\subsection{Morphology of blends: localization of JHNPs and emulsification effect}

The as-prepared silica/PS JHNPs were then incorporated at four different levels (1.5, 3, 5 and 10 phr) into PS/PA6 80/20 blends, using a twin-screw mini extruder. Extrusion temperature and screw speed were kept constant at $250^{\circ} \mathrm{C}$ and $120 \mathrm{rpm}$, respectively. After introduction of all components, time of mixing was fixed at $4 \mathrm{~min}$ under nitrogen flow. To investigate morphologies of nanocomposites, the injected sample disks are collected at the end of rheological test and cryo-fractured for scanning electron microscopy (SEM) observations. To evaluate quantitatively the emulsification effect using laser granulometer particle size analyzer, the PA6 nodules were collected using selective dissolution of the PS matrix in THF solvent.

Fig. 5 shows macroscopic views of three different PS/PA6/JHNPs 

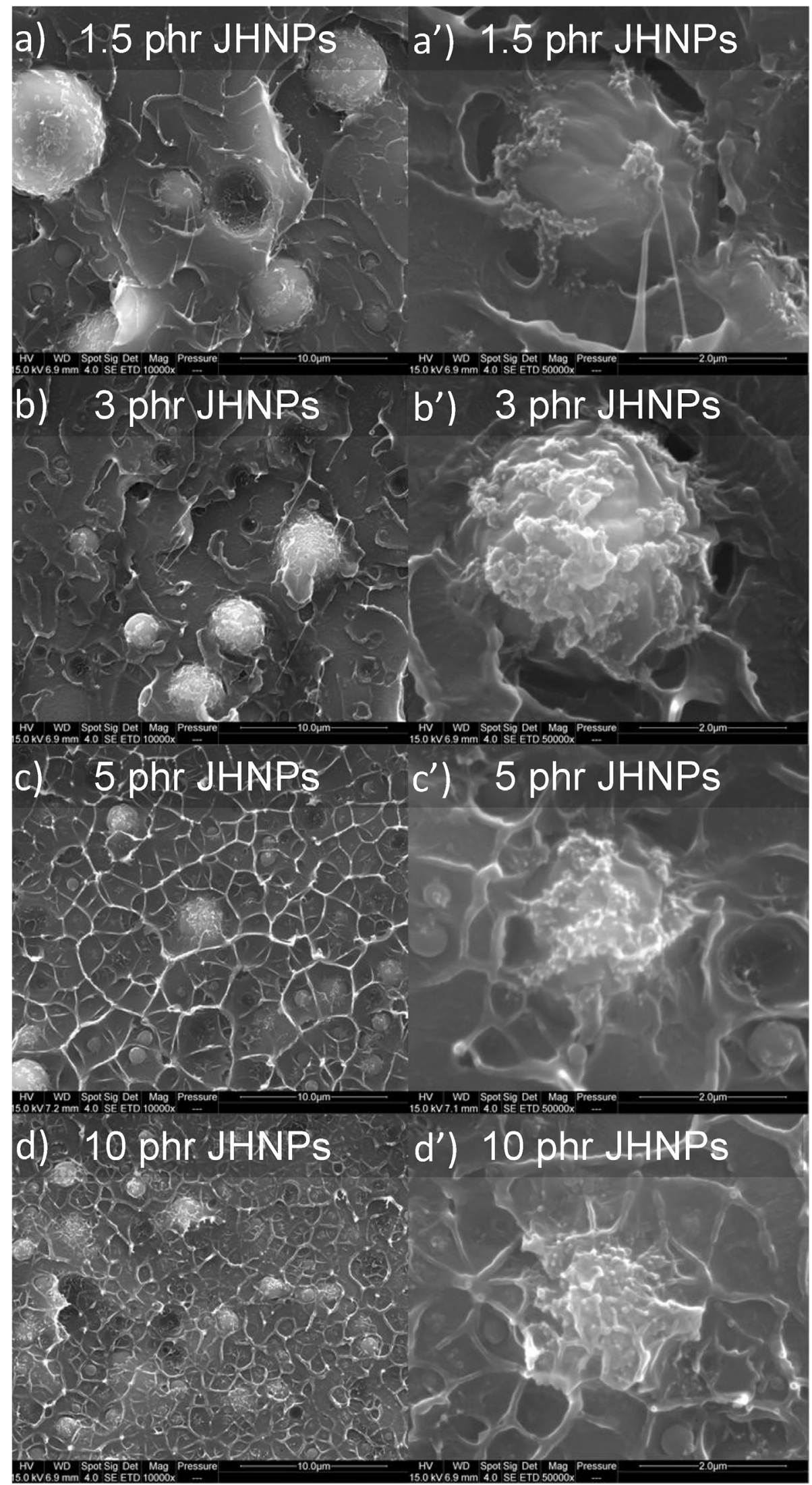

Fig. 7. SEM images of cryo-fractured PS/PA/JHNPs nanocomposite filled with $\left(a-a^{\prime}\right) 1.5,\left(b-b^{\prime}\right) 3,\left(c-c^{\prime}\right) 5$ and $\left(d-d^{\prime}\right) 10$ phr of JHNPs. Scale bar is $10 \mu \mathrm{m}$ for column $(a-d)$ and $2 \mu \mathrm{m}$ for column $\left(a^{\prime}-d^{\prime}\right)$. 


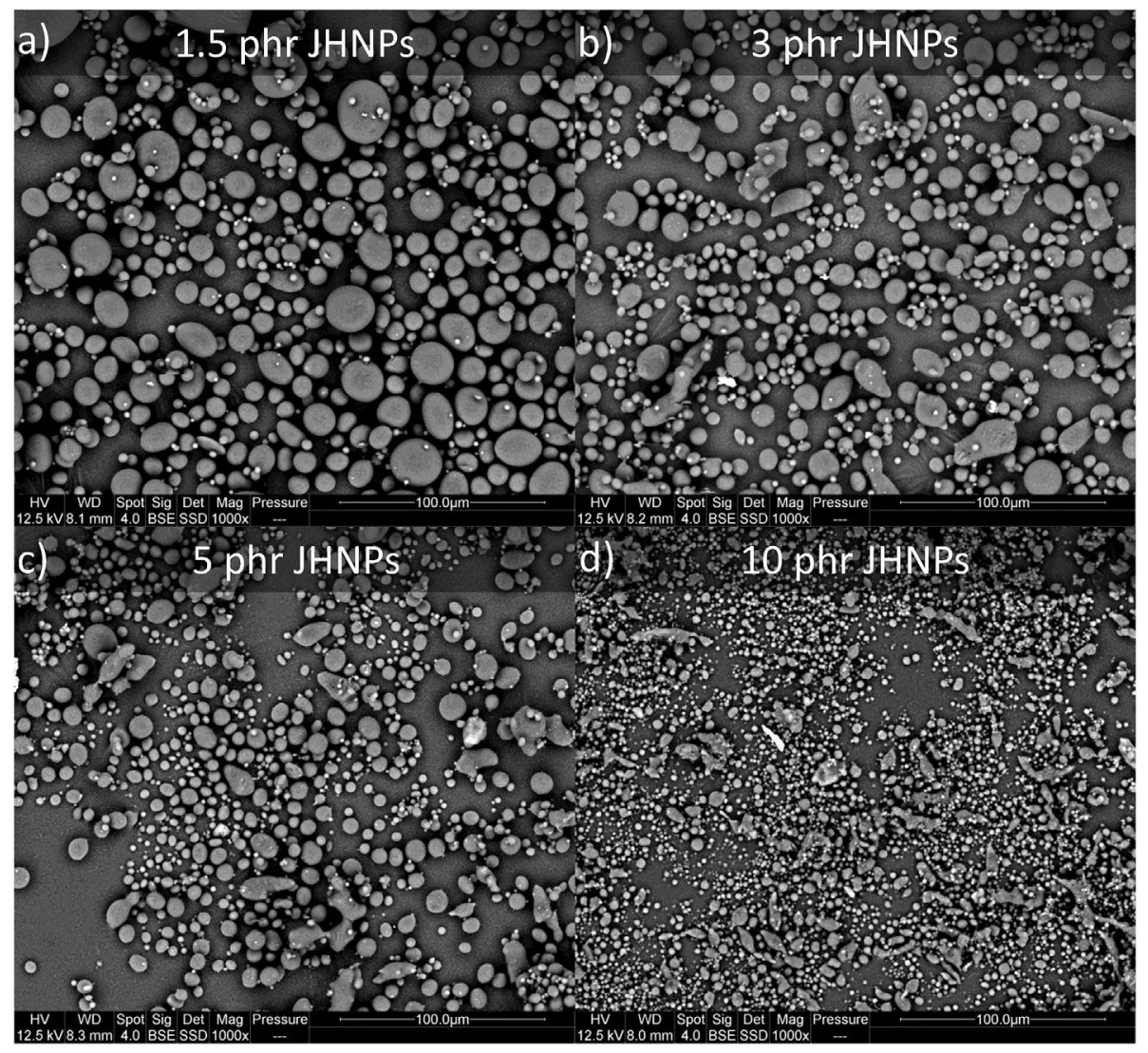

Fig. 8. SEM images of extracted PA6 nodules with (a) 0 , (b) 1.5, (c) 3 and (d) 10 phr of JHNPs at the end of rheological tests.

blends ( $0,1.5$ and 3 phr of JHNPs). For the neat blend, big spherical nodules of PA6 are observed, due to relaxation of elongated nodules and coalescence during the rheological test (Fig. 5a). When $1.5 \mathrm{phr}$ of JHNPs are incorporated a few nodules are still visible (Fig. 5b) whereas for the blend with $3 \mathrm{phr}$ of JHNPs no more nodules are visible (Fig. 5c) at the current observation scale. Moreover, we assume that the percolation threshold of the system is in the 3-5 phr range. In fact, blends with higher content of JHNPs (i.e. 5 and 10

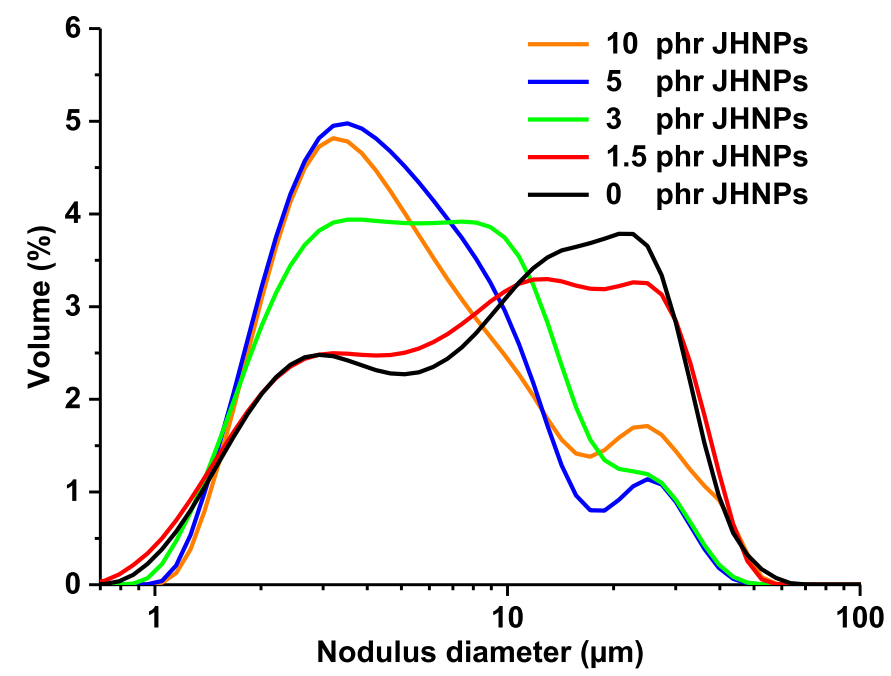

Fig. 9. Size distribution of PA6 nodules measured by laser diffraction particle size analyzer. phr) contain large agglomerates of JHNPs (see white circles in Fig. S3). To investigate the localization of JHNPs, a focus on the nanocomposite containing $1.5 \mathrm{phr}$ of JHNPs is displayed in the Fig. 6. The surface of the PA6 nodule appears partly covered by JHNPs (white circle in Fig. 6a). When PA6 nodules are ejected during cryo-fracture, the presence of JHNPs at the interface between the PA6 nodules and the PS matrix induces footprints in the PS matrix (see dotted white circle in Fig. 6a). To analyze more precisely the surface of PA6 nodules, selective extraction was

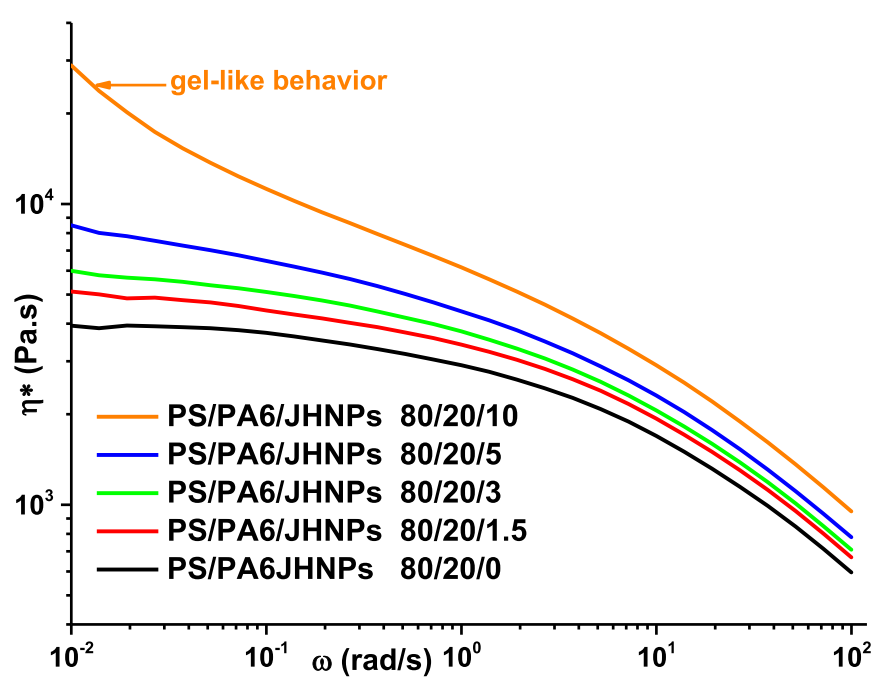

Fig. 10. Complex viscosity curves of blends from 100 to $0.01 \mathrm{rad} / \mathrm{s}$ at $230{ }^{\circ} \mathrm{C}$. 


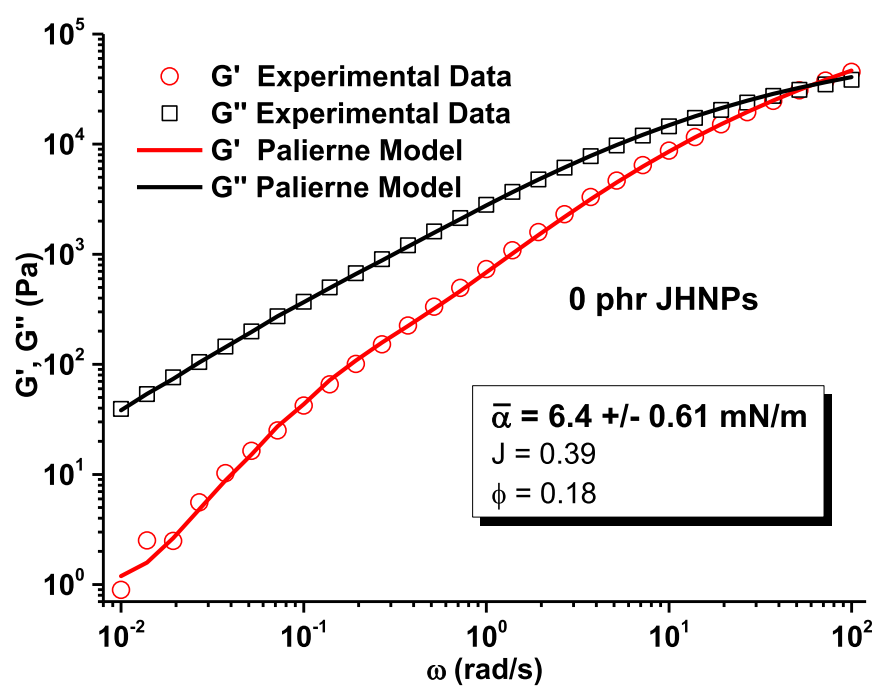

Fig. 11. Evaluation of interfacial tensions at $230{ }^{\circ} \mathrm{C}$ via optimization of Palierne model in the case of unfilled PS/PA6 80/20 system.

performed. An example of an isolated PA6 nodule collected using this method is shown in Fig. 6 b. Despite the three washing/ centrifugation cycles in THF to remove the entire PS matrix, the residual presence of JHNPs (white arrows in Fig. 6b) attests of good adhesion between the silica part of JHNPs and the PA6 nodule surface. To prove once again that JHNPs are localized at the surface of PA6 nodules, SEM-EDX analysis was performed. Silicon peak appears when pointed into supposed JHNPs whereas no more silicon peak is observed when a clear zone (without JHNPs) of the PA6 nodule surface is scanned (see Fig. S4).

The emulsification effect of these JHNPs at the interface was then investigated by SEM and laser diffraction particle size analyzer. SEM images of Fig. 7 allow to evaluate first, the decrease of size distribution as a function of JHNPs content (Fig. 7a-d) and second, the progressive recovery of PA6 surface nodules by JHNPs (Fig. $7 \mathrm{a}^{\prime}-\mathrm{d}^{\prime}$ ). This emulsification effect is easier to appreciate with the SEM images of extracted PA6 nodules in the Fig. 8. In order to precisely characterize the real size distribution of the dispersed phases, the extracted PA6 nodules after dissolution of the PS matrix, are analyzed using laser diffraction particle size analyzer. The size distribution curves of the different nanocomposites are displayed in Fig. 9. These results confirm once again that the incorporation of Janus nanoparticles decreases the volume average diameter of the PA6 nodules, known as emulsification effect. Indeed, this localization of Janus NPs suppresses the PA6 dispersed phase coalescence, leading to a decrease of the PA6 dispersed phase size. Fig. 9 highlights bimodal nodules distributions, especially for blends with low content of JHNPs ( 0 and $1.5 \mathrm{phr}$ ). As explained in our previous study on PS/PA6 blends filled with modified silica nanoparticles [31], this bimodal distribution is due to the initial presence of both spherical and elongated PA6 nodules. Indeed, elongated dispersed phases are generated in the skin polymer layer during both, extrusion and/or injection molding process. These elongated nodules then relax in big spherical ones during rheological tests. It must be noticed that

Table 1

Adjusted volume fraction and interfacial tension for PS/PA6/JHNPs blends.

\begin{tabular}{llll}
\hline PS/PA6 80/20 & JHNPs 0 phr & JHNPs 1.5 phr & JHNPs 3 phr \\
\hline$\Phi_{\text {adjust }}$ & 0.18 & 0.24 & 0.28 \\
$\bar{\alpha}(\mathrm{mN} / \mathrm{m})$ & $6.4 \pm 0.6$ & $4.5 \pm 0.48$ & $1.5 \pm 0.15$ \\
\hline
\end{tabular}

this bimodal distribution is almost no longer visible with 3, 5 and 10 phr of JHNPs. This is due to a lower polydispersity of the dispersed phase size when increasing the amount of JHNPs. The decreasing of the dispersed phase size is visible until a threshold of 5 phr of JHNPs associated to the saturation of the interface [39] and/or the formation of a solid network.

\subsection{Rheological behavior and interfacial tensions}

The last objective of this paper is to demonstrate that the emulsification effect is the consequence of the diminution of interfacial tension of blend when JHNPs are present at the interface.

As shown in Fig. 10, the complex viscosity of blends increases as a function of JHNPs content over the entire frequency range. In the case of nanocomposites with relatively high content of JHNPs (i.e 5 and $10 \mathrm{phr}$ ) no more Newtonian plateau at low shear rates are observed. A typical gel-like behavior (yield stress behavior) at low frequency is observed for the blend filled with $10 \mathrm{phr}$ of JHNPs (see Fig. 10). This phenomenon is usually attributed to the formation of solid network of nanofillers (percolation) through the material [27].

The interfacial tension $\bar{\alpha}$ is found to be the solution of the minimization problem described by Eq. (4). For all the formulations, around 10 iterations are necessary for to the convergence of this
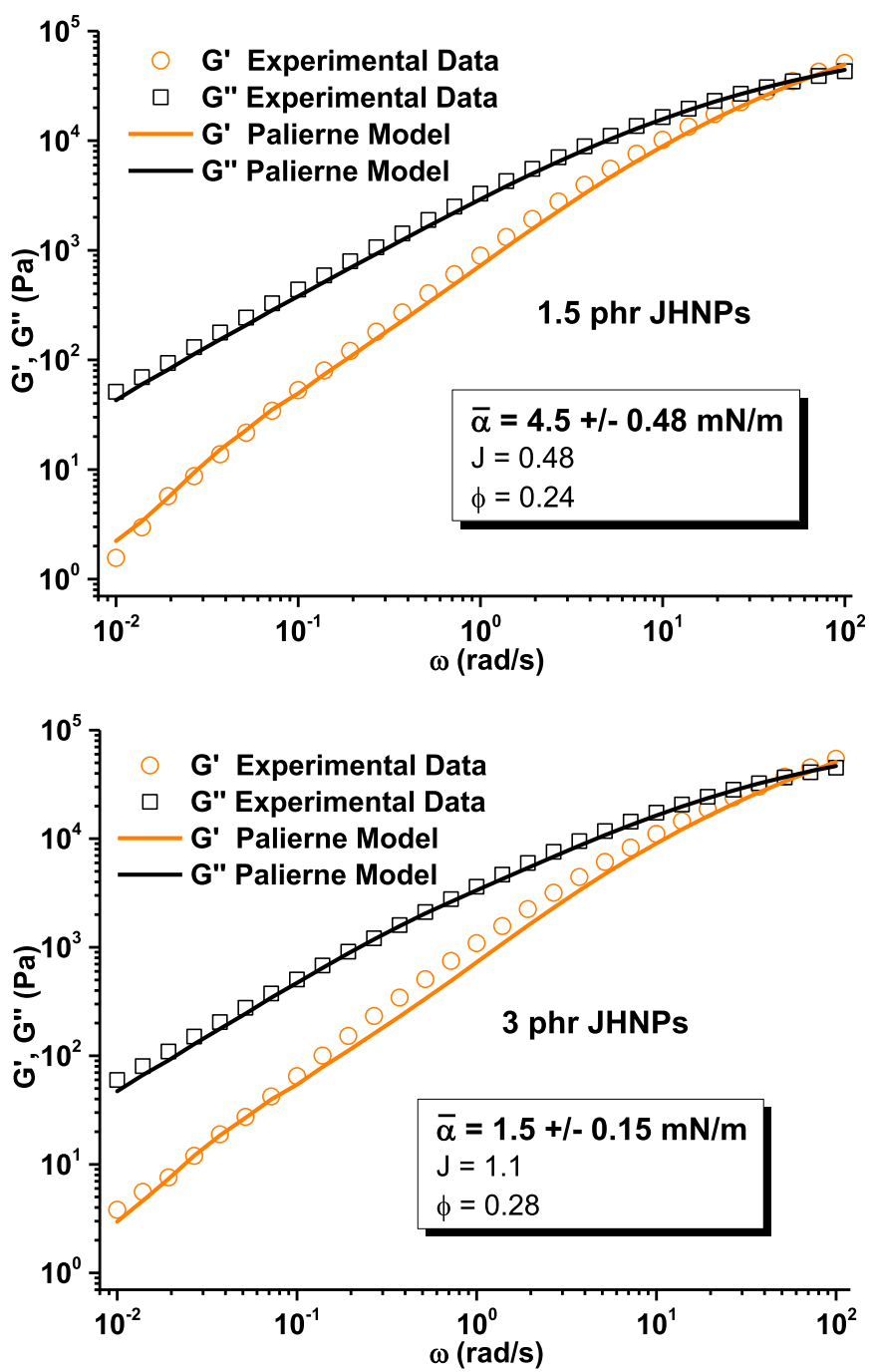

Fig. 12. Evaluations of effective interfacial tensions at $230{ }^{\circ} \mathrm{C}$ via optimization of Palierne model of nanocomposites filled with (up) 1.5 and (down) 3 phr of JHNPs. 
well-posed problem, independently of the interfacial tension initial value. We first evaluated the interfacial tension of the neat PS/PA6 blend. Because of the bimodal distribution of blends, the real size distributions of sample (Fig. 9) are considered for the Palierne model according to Equations (1) and (2). Fig. 11 shows the $\mathrm{G}^{\prime}$ (open circles) and $G^{\prime \prime}$ (open squares) experimental data points for the unfilled blend. The best fit of the Palierne model using the interfacial tension $\alpha$ as fitting parameter, was found for $\bar{\alpha}=6.4 \mathrm{mN} / \mathrm{m}$ and is also plotted in the Fig. 11. This evaluation is consistent with the results of Xing et al. [13]. These authors found with the same method an interfacial tension of $7.1 \mathrm{mN} / \mathrm{m}$ for a similar PS/PA6 80/ 20 system at $230{ }^{\circ} \mathrm{C}$.

In the case of polymer blends compatibilized with nanoparticles it is necessary to take into account the presence of JHNPs at the interface and their impact on the rheological response of the nanocomposite. The presence of the JHNPs at the PA6 nodule surface leads to the increase of their volume fraction. To use the Palierne model, an apparent volume fraction corresponding to the JHNPs/PA6 combination is determined. This volume fraction is adjusted using the Kerner model [27,28] (Palierne model with a zero interfacial tension value) at high frequency. Indeed, the Palierne model is not sensitive to interfacial tension values at high frequencies (see Fig. S5). Adjusted volume fractions ( $\left.\Phi_{\text {adjust }}\right)$ are therefore obtained for blends with 1.5 and $3 \mathrm{phr}$ of JHNPs (Table 1). The formation of spherical caps of nanoparticles around the PA6 nodules could explain the increase in the adjusted fractions as a function of JHNPS content. Due to the presence of JHNPs agglomerates for the blends with 5 and $10 \mathrm{phr}$, the adjusted volume fraction cannot be obtained and the corresponding interfacial tensions were not evaluated.

In a second step the interfacial tension is evaluated using the Palierne model with the previous adjusted volume fractions. Fig. 12 shows the best fit obtained for blends with 1.5 and 3 phr of JHNPs according to the optimization method described above. The Palierne predictions are in perfect agreements with experimental results. The associated interfacial tensions (see Table 1) decrease as the quantity of JHNPs increases. The interfacial tension of $6.4 \mathrm{mN} / \mathrm{m}$ determined for unfilled blend decreases to 4.5 and $1.5 \mathrm{mN} / \mathrm{m}$ for 1.5 and $3 \mathrm{phr}$ of JHNPs, respectively. This decrease confirms the compatibilizing ability of the Janus nanoparticles. These results prove that due to their amphiphilic character the JHNPs act as compatibilizing agents by reducing the interfacial tension of blend.

\section{Conclusions}

For the first time, Janus hybrid nanoparticles (JHNPs) composed of silica and polystyrene were incorporated into melted PS/PA6 immiscible polymer blend. We demonstrated the amphiphilic character of the home-made JHNPs via elemental analysis. Then, the JHNPs were introduced into polymers at the melted state and their selective dispersion at the interface of PS/PA6 system was observed using electron microscopy. This interfacial adsorption is due to the amphiphilic character of JHNPs. A strong emulsifying effect of the JHNPs was observed. A rheological method was used to prove that this morphology refinement is a consequence of the reduction of the interfacial tension due to the presence of JHNPs at the interface. This rheological method combines the linear viscoelastic measurements with the use of Palierne model to evaluate interfacial tensions of nanocomposites. The results show a significant reduction of the effective interfacial tensions from $6.5 \mathrm{mN} / \mathrm{m}$ for the neat blend to $1.5 \mathrm{mN} / \mathrm{m}$ for the nanocomposite filled with 3 phr of JHNPs. Ongoing challenge is to scale-up the quantities of synthetized JHNPs in order to study the effect on mechanical properties and compare it to usual compatibilizers.

\section{Acknowledgment}

The authors wish to thank Clément Gaussorgues, Angélique De Neiva and Benjamin Gallard for technical support. Patricia Beaunier for HRTEM-EDX analysis, professors Elodie Bourgeat-Lami, JeanFrançois Hochepied and Edith Peuvrel-Disdier for helpful discussions.

\section{Appendix A. Supplementary data}

Supplementary data related to this article can be found at http:// dx.doi.org/10.1016/j.polymer.2016.02.044.

\section{References}

[1] C. Koning, M. van Duin, C. Pagnouille, R. Jerome, Strategies for compatibilization polymer blends, Prog. Polym. Sci. 23 (1998) 707-757.

[2] R. Sonnier, A. Taguet, S. Rouif, Modification of polymer blends by E-beam and gamma-irradiation, Funct. Polym. Blends Synth. Prop. Perform. (2012) 261-304.

[3] A. Walther, K. Matussek, A.H.E. Müller, Engineering nanostructured polymer blends with controlled nanoparticle location using Janus particles, ACS Nano 2 (2008) 1167-1178, http://dx.doi.org/10.1021/nn800108y.

[4] A. Taguet, P. Cassagnau, J.-M. Lopez-Cuesta, Structuration, selective dispersion and compatibilizing effect of (nano)fillers in polymer blends, Prog. Polym. Sci. 39 (2014) 1526-1563, http://dx.doi.org/10.1016/j.progpolymsci.2014.04.002.

[5] T. Kwon, T. Kim, F.B. Ali, D.J. Kang, M. Yoo, J. Bang, et al., Size-controlled polymer-coated nanoparticles as efficient compatibilizers for polymer blends, Macromolecules 44 (2011) 9852-9862, http://dx.doi.org/10.1021/ ma2020134.

[6] F. Fenouillot, P. Cassagnau, J.-C. Majesté, Uneven distribution of nanoparticles in immiscible fluids: morphology development in polymer blends, Polymer 50 (2009) 1333-1350, http://dx.doi.org/10.1016/j.polymer.2008.12.029.

[7] M. Sumita, K. Sakata, S. Asai, K. Miyasaka, H. Nakagawa, Dispersion of fillers and the electrical conductivity of polymer blends filled with carbon black, Polym. Bull. 25 (1991) 265-271.

[8] R. Bahrami, T.I. Löbling, A.H. Gröschel, H. Schmalz, A.H.E. Müller, V. Altstädt, The impact of janus nanoparticles on the compatibilization of immiscible polymer blends under technologically relevant conditions, ACS Nano (2014) 11, http://dx.doi.org/10.1021/n502662p.

[9] K.C. Bryson, T.I. Löbling, A.H.E. Müller, T.P. Russell, R.C. Hayward, Using Janus nanoparticles to trap polymer blend morphologies during solventevaporation-induced demixing, Macromolecules 48 (2015) 4220-4227, http://dx.doi.org/10.1021/acs.macromol.5b00640.

[10] S. Weiss, D. Hirsemann, B. Biersack, M. Ziadeh, A.H.E. Müller, J. Breu, Hybrid Janus particles based on polymer-modified kaolinite, Polymer 54 (2013) 1388-1396, http://dx.doi.org/10.1016/j.polymer.2012.12.041.

[11] M.A. Fernandez-Rodriguez, Y. Song, M.Á. Rodríguez-Valverde, S. Chen, M.A. Cabrerizo-Vilchez, R. Hidalgo-Alvarez, Comparison of the interfacial activity between homogeneous and Janus gold nanoparticles by pendant drop tensiometry, Langmuir 30 (2014) 1799-1804, http://dx.doi.org/10.1021/ la404194e.

[12] S.H. Anatasiadis, Interfacial tension in binary polymer blends and the effects of copolymers as emulsifying agents, Adv. Polym. Sci. 238 (2011) 179-269.

[13] P. Xing, M. Bousmina, D. Rodrigue, M.R. Kamal, Critical experimental comparison between five techniques for the determination of interfacial tension in polymer blends: model system of polystyrene/polyamide-6, Macromolecules 33 (2000) 8020-8034, http://dx.doi.org/10.1021/ma000537x.

[14] M. Kontopoulou, Y. Liu, J.R. Austin, J.S. Parent, The dynamics of montmorillonite clay dispersion and morphology development in immiscible ethylene-propylene rubber/polypropylene blends, Polymer 48 (2007) 4520-4528, http://dx.doi.org/10.1016/j.polymer.2007.05.068.

[15] L. Elias, F. Fenouillot, J.C. Majesté, P. Alcouffe, P. Cassagnau, Immiscible polymer blends stabilized with nano-silica particles: rheology and effective interfacial tension, Polymer 49 (2008) 4378-4385, http://dx.doi.org/10.1016/ j.polymer.2008.07.018.

[16] J.S. Hong, Y.K. Kim, K.H. Ahn, S.J. Lee, C. Kim, Interfacial tension reduction in PBT/PE/clay nanocomposite, Rheol. Acta 46 (2007) 469-478, http:// dx.doi.org/10.1007/s00397-006-0123-1.

[17] J.E. Palierne, Linear rheology of viscoelastic emulsions with interfacial tension, Rheol. Acta 214 (1990) 204-214.

[18] H. Gramespacher, J. Meissner, Interfacial tension between polymer melts measured by shear oscillations of their blends, J. Rheol. 36 (1992) 1127, http:// dx.doi.org/10.1122/1.550304.

[19] M. Bousmina, R. Muller, Linear viscoelasticity in the melt of impact PMMA. Influence of concentration and aggregation of dispersed rubber particles, J. Rheol. 37 (1993) 663, http://dx.doi.org/10.1122/1.550389.

[20] D. Graebling, A. Benkira, Y. Gallot, R. Muller, Dynamic viscoelastic behaviour of polymer blends in the melt-experimental results for PDMS/POE-DO, PS/ PMMA and PS/PEMA blends, Eur. Polym. J. 30 (1994) 301-308, http:/ 
dx.doi.org/10.1016/0014-3057(94)90290-9.

[21] M. Bousmina, Rheology of polymer blends: linear model for viscoelastic emulsions, Rheol. Acta 38 (1999) 73-83, http://dx.doi.org/10.1007/ s003970050157.

[22] C. Lacroix, M. Bousmina, P.J. Carreau, B.D. Favis, A. Michel, Properties of PETG/ EVA blends: 1. Viscoelastic, morphological and interfacial properties, Polymer 37 (1996) 2939-2947, http://dx.doi.org/10.1016/0032-3861(96)89389-X.

[23] D. Graebling, R. Muller, J.F. Palierne, Linear viscoelastic behavior of some incompatible polymer blends in the melt. Interpretation of data with a model of emulsion of viscoelastic liquids, Macromolecules 26 (1993) 320-329, http://dx.doi.org/10.1021/ma00054a011.

[24] N. Mekhilef, P.J. Carreau, B.D. Favis, P. Martin, A. Ouhlal, Viscoelastic properties and interfacial tension of polystyrene-polyethylene blends, J. Polym. Sci. Part B Polym. Phys. 38 (2000) 1359-1368, http://dx.doi.org/10.1002/(SICI)10990488(20000515)38, 10<1359::AID-POLB130>3.0.CO;2-D.

[25] W.-R. Jiang, R.-Y. Bao, W. Yang, Z.-Y. Liu, B.-H. Xie, M.-B. Yang, Morphology, interfacial and mechanical properties of polylactide/poly(ethylene terephthalate glycol) blends compatibilized by polylactide-g-maleic anhydride, Mater. Des. 59 (2014) 524-531, http://dx.doi.org/10.1016/ j.matdes.2014.03.016.

[26] A. Asadinezhad, A. Yavari, S.H. Jafari, H.A. Khonakdar, F. Böhme, Description of the dynamic moduli of poly(trimethylene terephthalate)/polyamide-12 blends in molten state, Polym. Eng. Sci. 45 (2005) 1401-1407, http:// dx.doi.org/10.1002/pen.20413.

[27] L. Elias, F. Fenouillot, J.C. Majeste, P. Cassagnau, Morphology and rheology of immiscible polymer blends filled with silica nanoparticles, Polymer 48 (2007) 6029-6040, http://dx.doi.org/10.1016/j.polymer.2007.07.061.

[28] E.H. Kerner, The elastic and thermo-elastic properties of composite media, Proc. Phys. Soc. Sect. B 69 (1956) 808-813, http://dx.doi.org/10.1088/0370$1301 / 69 / 8 / 305$

[29] G.I. Taylor, The formation of emulsions in definable fields of flow, Proc. R. Soc. A Math. Phys. Eng. Sci. 146 (1934) 501-523, http://dx.doi.org/10.1098/ rspa.1934.0169.

[30] G.I. Taylor, The viscosity of a fluid containing small drops of another fluid, Proc. R. Soc. A Math. Phys. Eng. Sci. 138 (1932) 41-48, http://dx.doi.org/ 10.1098/rspa.1932.0169.
[31] T. Parpaite, B. Otazaghine, A. Taguet, R. Sonnier, A.S. Caro, J.M. Lopez-Cuesta Incorporation of modified Stöber silica nanoparticles in polystyrene/ polyamide- 6 blends: coalescence inhibition and modification of the thermal degradation via controlled dispersion at the interface, Polymer 55 (2014) 2704-2715, http://dx.doi.org/10.1016/j.polymer.2014.04.016.

[32] W. Lu, M. Chen, L. Wu, One-step synthesis of organic-inorganic hybrid asymmetric dimer particles via miniemulsion polymerization and functionalization with silver, J. Colloid Interface Sci. 328 (2008) 98-102, http:// dx.doi.org/10.1016/j.jcis.2008.08.043.

[33] J. Parvole, I. Chaduc, K. Ako, O. Spalla, A. Thill, S. Ravaine, et al., Efficient synthesis of snowman- and dumbbell-like silica/polymer anisotropic heterodimers through emulsion polymerization using a surface-anchored cationic initiator, Macromolecules 45 (2012) 7009-7018, http://dx.doi.org/10.1021/ ma301355g.

[34] Y. Yin, S. Zhou, B. You, L. Wu, Facile fabrication and self-assembly of polystyrene-silica asymmetric colloid spheres, J. Polym. Sci. Part A Polym. Chem. 49 (2011) 3272-3279, http://dx.doi.org/10.1002/pola.24762.

[35] J. Kim, R.J. Larsen, D.A. Weitz, Synthesis of nonspherical colloidal particles with anisotropic properties, J. Am. Chem. Soc. 128 (2006) 14374-14377, http://dx.doi.org/10.1021/ja065032m.

[36] W. Qiang, Y. Wang, P. He, H. Xu, H. Gu, D. Shi, Synthesis of asymmetric inorganic/polymer nanocomposite particles via localized substrate surface modification and miniemulsion polymerization, Langmuir 24 (2008) 606-608, http://dx.doi.org/10.1021/la703607s.

[37] A. Perro, S. Reculusa, F. Pereira, M.-H. Delville, C. Mingotaud, E. Duguet, et al., Towards large amounts of Janus nanoparticles through a protection-deprotection route, Chem. Commun. (2005) 5542, http://dx.doi.org 10.1039/b507486j.

[38] Y. Wang, H. Xu, Y. Ma, F. Guo, F. Wang, D. Shi, Facile one-pot synthesis and morphological control of asymmetric superparamagnetic composite nanoparticles, Langmuir 27 (2011) 7207-7212, http://dx.doi.org/10.1021/ la200749w.

[39] B.D. Favis, Phase size/interface relationships in polymer blends: the emulsification curve, Polymer 35 (1994) 1552-1555, http://dx.doi.org/10.1016/ 0032-3861(94)90358-1. 